\title{
A Scalable Distributed Positioning System Augmenting WiFi Technology
}

\author{
Stefano Maddio ${ }^{1}$, Member, IEEE, Marco Passafiume ${ }^{2}$, \\ Alessandro Cidronali ${ }^{3}$, Member, IEEE, Gianfranco Manes ${ }^{4}$, Senior Member, IEEE \\ \# Department of Information Engineering, University of Florence, V.S. Marta, 3, I-50139, Florence, Italy \\ ${ }^{1}$ stefano.maddiodunifi.it ${ }^{2}$ marco.passafiume@unifi.it ${ }^{3}$ alessandro.cidronali@unifi.it \\ ${ }^{4}$ gianfranco.manesdunifi.it
}

\begin{abstract}
This paper presents a distributed localization system for indoor environment based on a network of compact anchor nodes. The system is based on available low-cost commercial components and it is capable of standard WiFi connectivity at $2.45 \mathrm{GHz}$. The basic node is equipped with a switch-beam antenna, which is the enabling technology for a Direction of Arrival (DoA) estimation based on Maximum-Likelihood criteria. The adopted DoA estimation procedure is tolerant to noisy power measurements, making it suitable for low-cost effective solutions based on RSSI measurements.

The node is designed to be placed in an unobtrusive area for the movement of a mobile target node and is capable to operating as an independent anchor, but it is optimized to cooperate with other identical nodes to form a coordinated network of anchors, capable to monitor an extended indoor area.

An experimental characterization demonstrates the DoA performance of the single node.

Index Terms-Indoor positioning system, Switched beam antenna (SBA), Direction of arrival (DoA), Received Signal Strength Indicator (RSSI).
\end{abstract}

\section{INTRODUCTION}

There is a great interest around the potential impact of positioning systems for GPS-denied scenarios for their consequences in the scientific and the social community. The position awareness that comes from the localization procedure has great impact in various research field, especially in the field of Wireless Sensor Network (WSN), where the positional information can boost specific routing operations (adaptability, latency, throughput) of a network. The position awareness can be also exploited for a wide spectrum of context-aware applications which span from art monitoring to surveillance application, from asset management to indoor navigation and so on [1], [2].

Among the various localization techniques, the approaches based on Received Signal Strength Indicator (RSSI) present several advantages. RSSI is a coarse power measurement for radio signal which is commonly equipped in commercial transceivers, typically available to the user in the form of a digital data on a register.

RSSI measurements are already employed for range estimation based on channel propagation model, which is the first step of trilateration approach. Unfortunately the noisy characteristics of the radio channel results in high inaccuracies

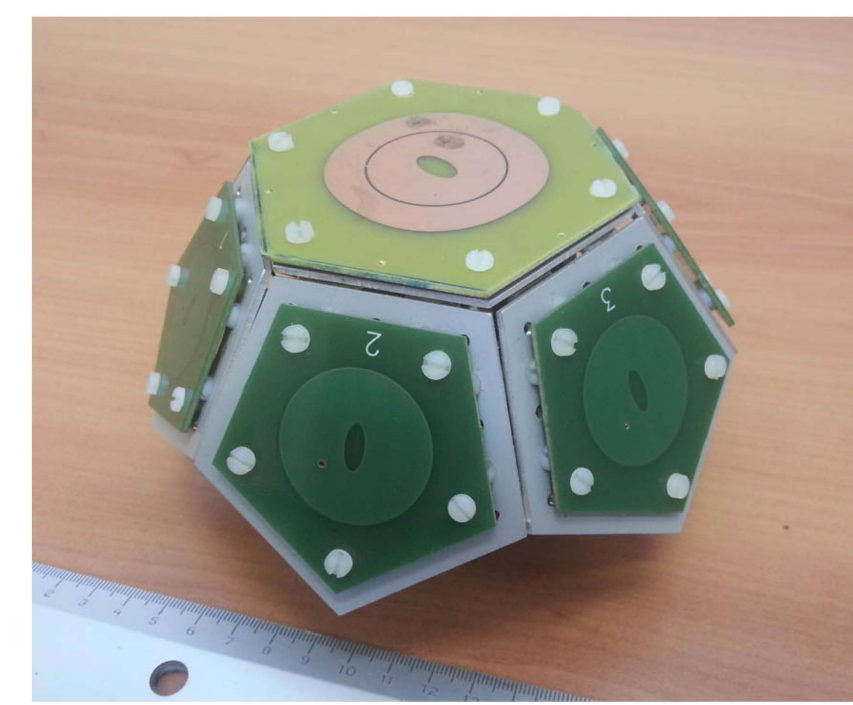

Fig. 1. The anchor node built around the Switched Beam Antenna.

of the positional information. RSSI measurements can also be employed for Direction of Arrival (DoA) estimation, which is a range-free approach. In this case the noise issue has reduced impact, and can be further reduced with adequate system architecture [3].

The key of DoA estimation is the Space Division Multiple Access (SDMA), the channel access method based on signal reception using directive antennas. A Switched Beam Antenna (SBA) is a specialized radio system capable to radiate $\mathrm{N}$ directional beam patterns, operating as a spatial multiplexer alternatively isolating the signal reception from specific angular regions. The impact of SBA on the communication layer can be tremendous [4], [5], [6].

With reference to Fig. 2, consider a target node $T$ which is in the communication range of two anchor nodes, $A$ and $B$. Upon the reception of radio signals from $T$ through the set of its radiation sectors, the node $A$ estimates the Direction of Arrival of the incoming message on the basis of a likelihood criterion driven by radio beam diversity. The DoA of the messages identifies the Line of Bearing ( $\mathrm{LoB}$ ) of $T$ respect to the local reference of the anchor $A$. The more discriminated is the beam set of $A$, the more reliable is the estimate of the LOB. 


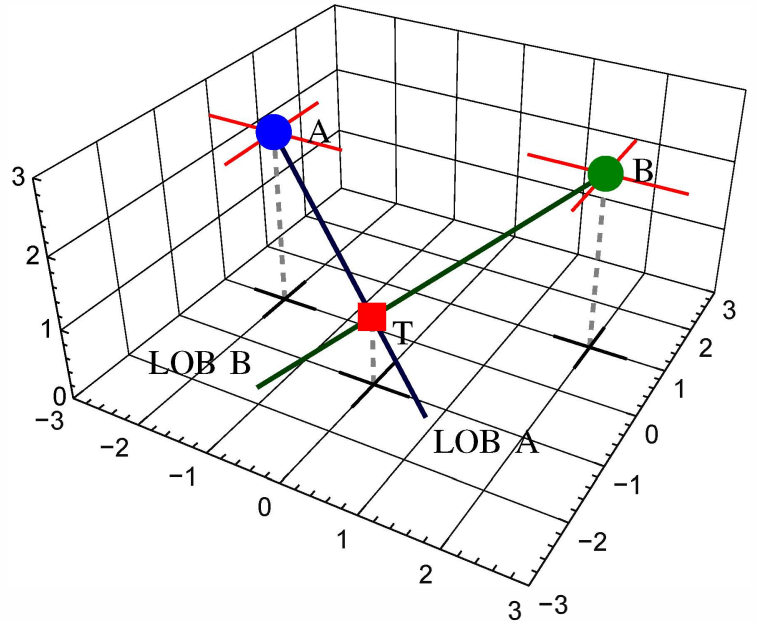

Fig. 2. Scheme for distributed localization. Each anchor identify a Line of Bearing and a set of lines resolves in the absolute target position.

The same procedure is carried by the anchor $\mathrm{B}$, which identify a second B-centered LOB. The triangulation of the two LoB's results in the estimation of the absolute position using simple trigonometric relationships. As for the trilateration, a higher number of anchors means an over determined set of data, which results in a more noise tolerant estimation.

In this paper, a positioning system based on compact independent nodes is presented. In particular, the optimum design of the basic node is object of extensive investigations and validations. Each node acts as a specialized WiFi access point, equipped with standard Ethernet controller, capable to estimate the DoA of independent target nodes by exchanging radio messages. Since the RSSI is automatic retrieved during any packet exchange, the necessary information for the localization comes at a very low cost in terms of protocol resources. An eventual remote server, acting as the supervisor of the network activity, can coordinate and aggregate the data to synthesize the positional information with standard triangulation technique.

The key feature of the proposed system is the ability to offer a minimal positioning system based on low-cost commercial components which at the same time behaves as the unit of a more complex distributed localization system.

In order to explain our solution this paper is organized as follows: Section II describes the hardware of the single node, while section III is focused on the SBA, covering the various aspects of its design. Section IV describes the localization algorithm and finally section $\mathrm{V}$ presents the obtained localization performance of a single node. In Section VI some conclusions is been drawn, as well as future developments for the system.

\section{ARCHITECTURE OF THE SYSTEM}

Fig. 3 shows a photo of the hardware of the anchor node. The core of the node, on the right side of the photo, is CC2430 from Texas Instruments, an highly integrated RF SystemOn-Chip chip built in $0.18 \mu \mathrm{m}$ CMOS standard technology.
As a transceiver, this device is capable of $2.4 \mathrm{GHz}$ Direct Sequence Spread Spectrum operation and it is complaint with IEEE 802.15.4 protocol and suitable for the ZigBee operation, while the embedded 8-bit core is compatible with the high performance and efficient 8051 industrial controller. This device comes with an embedded in-system programmable flash memory of $128 \mathrm{~Kb}, 8 \mathrm{~Kb}$ RAM. These computational resources, though limited, are adequate for the distributed elaboration demanded to each node.

On the down side of the board the General Purpose Input/Output (GPIO) pins, which are employed to directly control the Switched Beam Antenna peripheral described in the following section (Section III), which is fed by the RF outport on the top side of the transceiver.

For our goals, the most important feature is the built-in RSSI module, always available to the micro and to the end user, which operates averaging the received signal energy over an 8 symbols periods $(128 \mu \mathrm{s})$ and returning this value in the form of a formatted data register, in accord with the IEEE protocol. This 8-bit data is related to the actual incoming power in $\mathrm{dBm}$ through an equation provided by the manufacturer. The RSSI resolution of the chosen device is only of $1 \mathrm{~dB}-$ a common performance for commercial transceivers of this class/price nevertheless this RSSI is adequate to serve as input data of the localization engine, consenting a convenient trade off between price and quality.

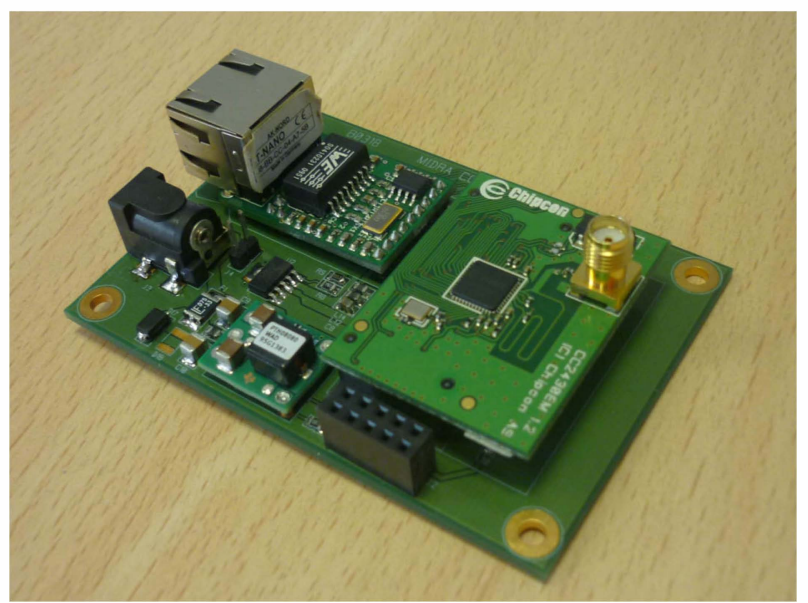

Fig. 3. Node main board. The CC2430, the Ethernet module, the power plug and the GI/O pins are visible.

The motherboard of the node is equipped with a an Ethernet controller, employed for all the communications between the anchor node and the remote supervisor. This system is suitable for low-power operation, being the power consumption of the SoC below $27 \mathrm{~mA}$ in either transmitting or receiving mode, while providing a wide supply voltage range $(2.0 \mathrm{~V}-3.6 \mathrm{~V})$. Very fast transition times from low-power modes $(0.3 \mu \mathrm{A}$ in stand-by mode and $0.5 \mu \mathrm{A}$ in power-down mode) to active mode enables ultra low average power consumption in low duty-cycle systems, making this device especially suitable for the applications with a low duty cycle. 


\section{SWitched BEAM ANTENNA}

A Switched Beam Antenna (SBA) is an antenna array fed by a Beam-Forming Network (BFN) and it is capable of a predetermined set of beams selected by an appropriate digital control [7]. BFN can be implemented at IF or RF level. The simplest form of SBA consists in set of antenna elements pointing toward different directions, fed by a Single Pole N Through (SPNT) RF switch.

The optimal antenna arrangement is the one with each element at its maximum gain where the other are in a lowgain, a condition which grants angular uncorrelated signals, which is the foundation of the likelihood approach [1]. At the same time the cumulative pattern, - i.e. the envelope of all the beams - should cover the entire area of interest in order to avoid null signal zones.

Antennas operating in Circular Polarization (CP) are of strong aid for indoor wireless communications, since they are capable to mitigate multi-path impairments in complex radio environments, making the RSSI data much less noisy [8]. In addition, $\mathrm{CP}$ antennas are indifferent to rotation around their axis, which relaxes the pointing issue, making them perfect candidate for the application in exam, since communication is possible regardless of the relative orientation. The improvement of CP for indoor positioning has been subject of researches [9].

The actual type and arrangement of the antenna elements has a great impact on the performance of the localization system (as explained in [1], [3], [10]).

\section{A. Antenna Element of type I}

Modal degeneration is an effective technique to design CP microstrip patch antennas. A quasi-symmetrical patch supports two orthogonal degenerated resonant modes [11], [12]. If the opportune detuning is imposed and the feed position is chosen as to excite both of them with the same intensity but in phase quadrature, the resultant field is circularly polarized. This perturbation mechanism can be applied to any resonant mode, being the actual type of the asymmetry a consequence of the specific resonant eigen-function shape [13].

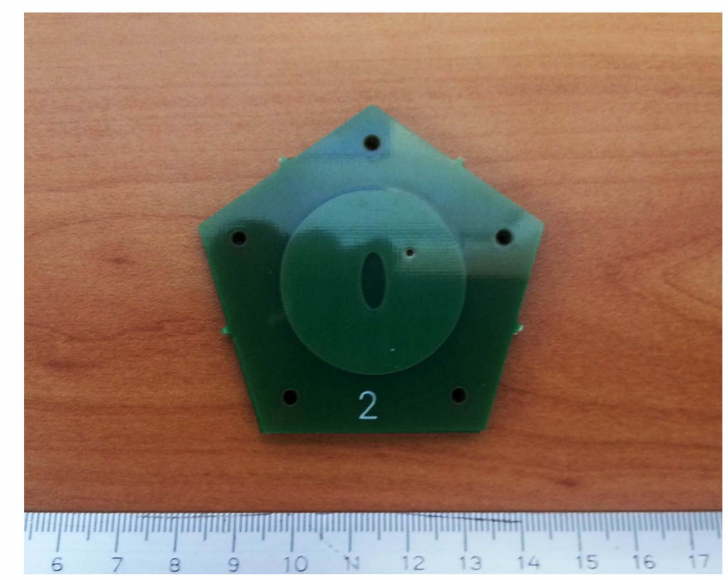

Fig. 4. Antenna element of type I.

A disc based antenna is the most suitable geometry for the SBA, thanks to its conformability to regular polygon shapes.

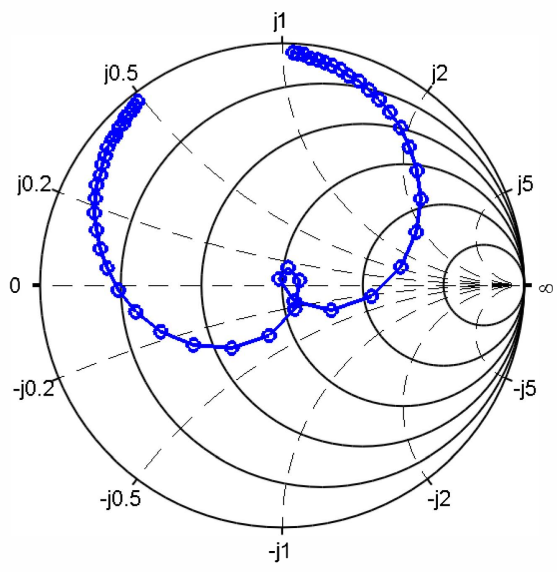

(a) Input impedance on the Smith Chart

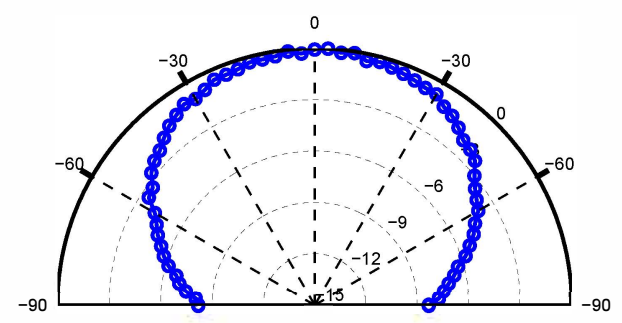

(b) Principal cut of the left hand pattern at the center frequency

Fig. 5. Measured performance of the Elliptical Slitted Disk Antenna.

A canonic disk working in its fundamental $T M_{11}$ mode is the source of a directional beam with its maximum in the boreside direction.

A direct way to degenerate the $T M_{11}$ mode and hence to obtain a directive $\mathrm{CP}$ radiator, is to employ a central elliptical cut as detuning element. With the adequate dimension of the ellipse, the frequency responses of the two modes, $T M_{11 e}$, $T M_{11 o}$, can be made overlapping, enabling the opportune combination for the degeneration.

Furthermore, the elliptical cut makes the patch a loop, consenting a further parameter for the control of the radiator performance. This geometry, named Elliptical Slitted Disk Antenna (ESDA), was already discussed by the author, [14], [15], and it was already employed for localization purposes [1], [7], so it won't be further discussed.

Fig. 4 depicts the optimal ESDA prototype, labeled as type I hereinafter, which is printed in common low-cost FR4 dielectric substrate $\left(\epsilon_{r}=4.4, h=1.6 \mathrm{~mm}\right)$, while Fig. 5 presents the relevant measured performance. The 3D gain pattern is a body of revolution, with section described by 5(b), and maximum values around

\section{B. Antenna Element of type II}

The same approach of the previous section can be applied to a disc operating in $T M_{21}$ mode. When operating in this mode, the patch antenna presents a conical beam pattern, sometimes 
described as a $2 D$ omni directional shape, with deep nulls in the upper and lower boreside direction and a maximum in the region around $35^{\circ}-70^{\circ}$ [12, pp. 346-349], [13, p. 135-149].

The Lame' shape, also known as Superellipse, is a generalization of the the disc geometry, suitable to excite the modal degeneration of $T M_{21}$ mode [16]. This curve is described in polar coordinates as

$$
\rho(\theta)=R \sqrt{(\cos \theta)^{\frac{2}{m}}+(\sin \theta)^{\frac{2}{m}}}
$$

where $m$ is parameter controlling the level of squareness i.e, with $m=1$ the disc reverts to the canonic shape, with $m=\infty$ tends to a square. As for the fundamental mode, the super-ellipse can host two detuned orthogonal modes $T M_{21 e}$, $T M_{21 o}$, physically similar to the unperturbed $T M_{21}$, with an opportune shape of the patch, i.e. with the opportune exponent.

As a disc patch antenna can be made annular keeping the same radiative performance, the same goes for a superelliptical loop. The dimensions of a $T M_{21}$ resonator are almost the double of the $T M_{11}$ resonator, and the elliptical cut of the latter gives another degree of freedom: thanks to the complementary of the patch geometries, it is possible to arrange a $T M_{11}$ and a $T M_{21}$ operating disc antennas in a concentric manner, with the second radiator developed as an annular ring surrounding the first one. The realized structure can be considered a two beams antenna, with the radiation pattern set by the fed input (Fig. 6).

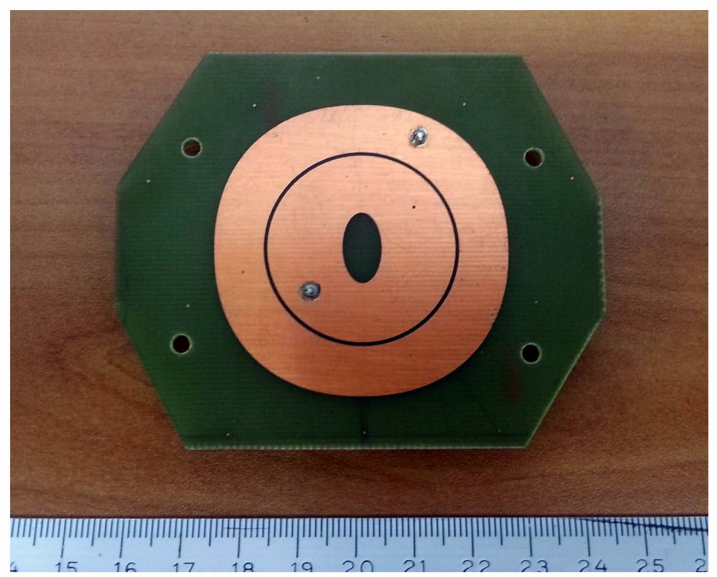

Fig. 6. Antenna element of type II. The two feeds, revealed by the solder points, control the actual beam shape.

Unfortunately, this arrangement reduces the maximum gain of the $T M_{21}$ mode. Nevertheless, this two beams antenna was already proposed and discussed by the authors, [16], demonstrating its localization ability even by itself, as an azimuth of arrival estimator based on couples of RSSI measurements. Fig. 7 presents the measured performance of this antenna element, labeled as type II, printed on the same common lowcost FR4 dielectric substrate of the previous element.

\section{Elements Arrangement}

The proposed system is intended to be placed on the ceiling of indoor environment with the theta reference downwards facing, therefore the expected coverage area is a circular region

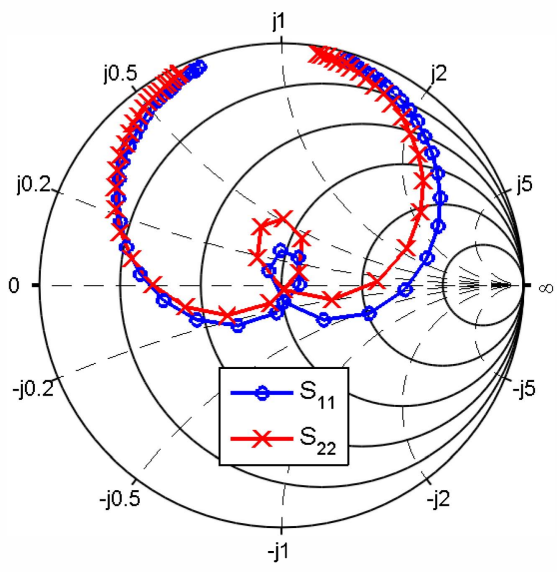

(a) Input impedances on the Smith Chart

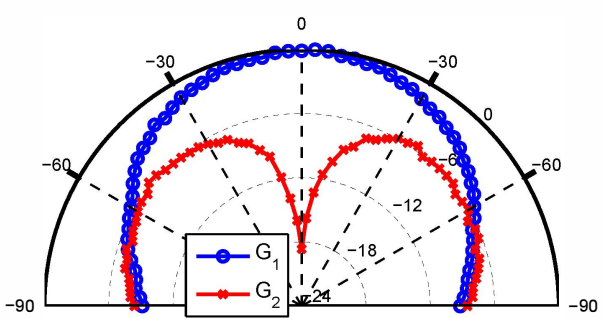

(b) Principal cut of the left hand pattern at the center frequency. The second mode pattern is normalized to the first mode maximum.

Fig. 7. Measured performance of the the two beams antenna element.

centered on the projection of the node, a unit cell of the eventual network. The placement of the anchor nodes and their constellation has great importances and deserve a tractation which is beyond the scope of this paper [17]. To meet the requirement of uniformity, and considering that the antenna elements show a cardioid-like pattern, the best arrangement for the SBA is the polyhedron depicted in Fig. 1, arranged on a light shell of aluminum. This shape, while not platonic, is the best trade-off between desired performance and physical constraints.

With reference of Fig. 8(a), the antenna elements labeled from \#2 to \#7 are of type I. they are arranged in a hexagonal manner, with the dihedral angle between them around $120^{\circ}$. The dihedral angle between \#2-\#7 and the top \#1 face is around to $115^{\circ}$. The complementary behavior of the type II antenna, in term of $\theta$ coordinate, makes it a perfect candidate for the top element of the SBA, namely the beams \#1 and \#8. The beam diversity is actuated by feeding one of the elements of the set with the HMC253QS24 GaAs SP8T non-reflective switch. The switch is characterized with a nominal insertion loss of $1.8 \mathrm{~dB}$ and an isolation exceeding $30 \mathrm{~dB}$ at $2.45 \mathrm{GHz}$, which is enough for the purpose of this work. The adoption of this kind of switch minimizes the interaction between the radiators, which is a plus for the device needs.

Supposing a Friis path-loss model, when placed at the height 


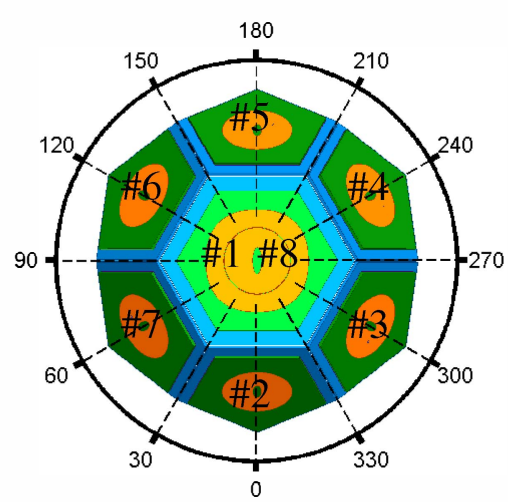

(a) Anchor reference

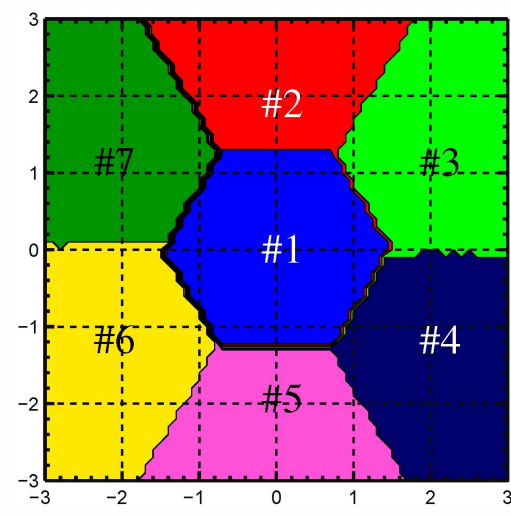

(b) Signal partition in seven areas

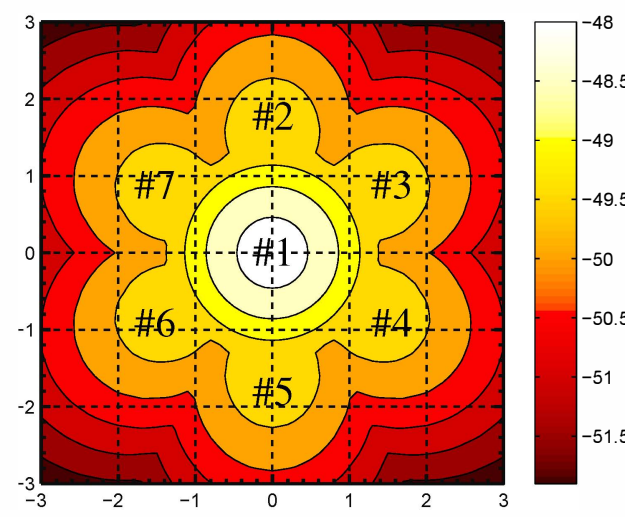

(c) Cumulative pattern

Fig. 8. Space partition and signal coverage of the proposed Switched Beam Antenna. In (a), the node is seen from above, with the polar reference of $\phi$ in evidence. The projection of (b) and (c) are obtained with the node hanging from the ceiling, therefore the different clockwise direction of the projections.

of 3 meter at the center of an environment like the one depicted in Fig. 2, the SBA projects the cumulative power distribution depicted in Fig. 8(c). In this model, the target is placed at the height of 1.25 meters, compatible with the position of a personal mobile device held by human hand. It is important to note that, while simple, this first-order model is not unrealistic thanks to the CP operation of the SBA which makes the secondary multi-path bounces very weak, making the model adequate. As a side note, the ability to partition the space can be considered a rough localization algorithm by itself, which will play a role in the following sections.

With reference to Fig. 8(b), the seven areas projected by the seven elements \#1 to \#7 are well identified, indicating an adequate angular discrimination behavior, while the cumulative pattern confirms the almost uniform coverage of the area, with a quite constant power ranging from $-52 \mathrm{dBm}$ to $-47 \mathrm{dBm}$. This solution is effective for the creation of a localization cell suitable for a space filling arrangement based on hexagonal lattice, which grants a uniform tiling for the area coverage.

\section{Pattern Characterization}

The SBA was subjected to measurement to derive the gain pattern. The accuracy of the pattern in numeric form has great impact on the localization performance [18]. The SBA gain was measured in a laboratory anechoic chamber with a Vector Network Analyzer (VNA) and an antenna rotor synchronized by a proper control software. The data were collected at the central frequency of $2.45 \mathrm{GHz}$, along the various cuts of the SBA identified by the reference depicted in Fig. 8(a).

Fig. 9 report the measurements taken for the cut $\theta=90^{\circ}$, which corresponds to the equatorial cut. The six side elements irradiate six equally spaced beams, with similar shapes. Although this is not the cut corresponding to the maximum radiation, the measured $\mathrm{HPBA}$ is around $80^{\circ}$, confirming the expected space multiplexing ability in the $\phi$ angular coordinate.

Fig. 10 reports one of the principal cut, the one starting from face \#2 passing through the faces \#1+\#8 to reach face \#5 - cut from $\phi=0^{\circ}$ to $\phi=180^{\circ}$ of Fig. 8(a). The figure highlights the

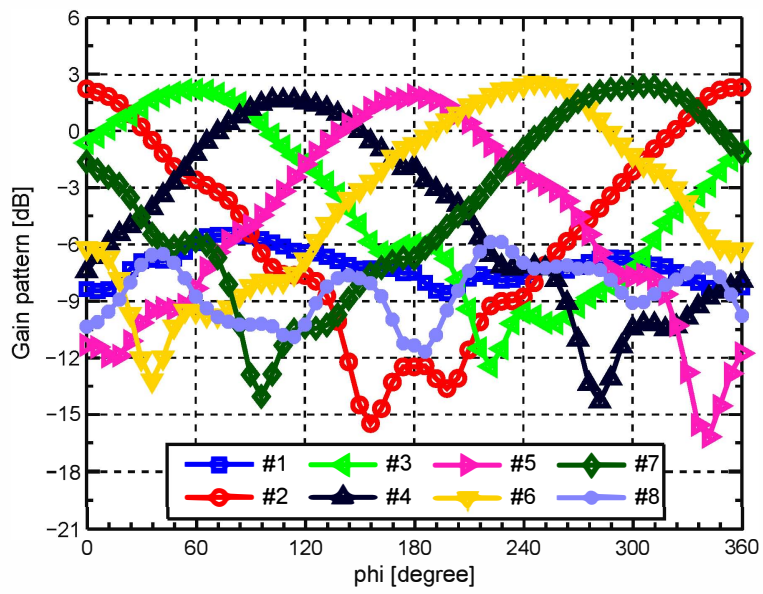

Fig. 9. Measurement of the gain cut corresponding to $\theta=90^{\circ}$ (equatorial cut).

space division along the $\theta$ coordinate, enforced by the shape of the beam \#1 and \#8, confirming the measurement of Fig. 7(b). Similar measurements were taken for all the principal and the intermediate cuts, which are not reported. The experimental characterization confirms almost the same behavior in each cut.

The far-field of the entire set, $\mathbf{G}(\theta, \phi)$, rebuilt using interpolating technique [19], [20], is an important input parameter of the localization engine described in the following section. The measurements and the consequent interpolation are limited to the upper hemisphere, which corresponds to the direction facing toward the floor, the only one where the target devices are supposed to operate. The rebuilt gain is stored in a $3 \mathrm{D}$ matrix of dimensions $N \times T \times P$, where $N=8$ is the number of SBA beams, while $T \times P$ is the dimension of the $(\theta, \phi)$ grid describing the half space of interest.

\section{LOCALIZATION ALGORITHM}

The proposed system is suitable for a large class of DoA estimator. The most efficient algorithms are the spectral-based ones, such as Multiple Signal Classification (MUSIC) [21]. 


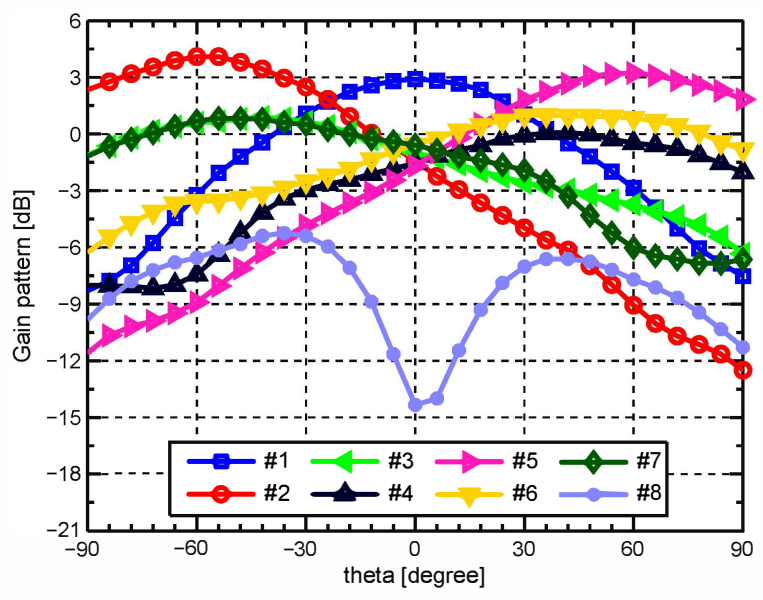

Fig. 10. Measurement of the gain cut corresponding to $\phi=0^{\circ}$ (negative semi-axis) and $\phi=180^{\circ}$ (positive semi-axis).

This algorithm estimates the $\operatorname{DoA}\left(\widehat{\theta}_{t}, \widehat{\phi}_{t}\right)$ on the basis of the spectral decomposition of the covariance matrix of the RSSI readings on the set of antenna elements. Considering a fast sequential reception for the channel coherence time, no assumptions on the propagation model are necessary, since the RSSI vector is taken as a synchronous array.

During the exchange of packets with the target - which enters in broadcast condition after a request message - an anchor node collects the RSSI vector $\mathbf{S}$ :

$$
\mathbf{S}=\mathbf{G}\left(\theta_{T}, \phi_{T}\right)+\mathbf{P}_{\mathbf{r x}}+\mathbf{n}
$$

where $\mathbf{G}\left(\theta_{T}, \phi_{T}\right)$ is the gain map for the DoA $\left(\theta_{T}, \phi_{T}\right)$, $\mathbf{P}_{\mathbf{r x}}$ is the receiving power impinging on the SBA in vector form and $n$ is an average Gaussian $\mathrm{N}$-dimensional noise. In this observation model, the actual gain of the target node is irrelevant, since all the SBA elements experiment the same impinging power, since the node is point-like for the involved distances, therefore $\mathbf{P}_{\mathbf{r x}}=\left[P_{r x}, P_{r x}, \ldots P_{r x}\right]$, with $P_{r x}=P_{t x}-P L$, with $P L$ Friis PathLoss. The CP operation mode of the antennas makes this simple observation model adequate.

It is worthwhile to remark that if more target broadcast their hello message, the anchor are capable to discriminates the source of the packets at firmware level, therefore the undesired RSSI can be discarded. In practice an anchor collects all RSSI data and store them in cycle buffer, with each data labeled with the proper source target.

Let $\mathbf{S}(k)$ be the vector of RSSI values sampled at discrete time step $k$, that is:

$$
\mathbf{S}[k]=\mathbf{S}\left(T_{0}+k \Delta t\right) .
$$

Starting from $K$ repeated readings, an estimation $\widehat{R}_{S S}$ of the correlation matrix $R_{S S}$ of the signals is given by:

$$
\widehat{R}_{S S}=E\left[\mathbf{S}[k] \mathbf{S}[k]^{\top}\right]=\sum_{k=i}^{K} \mathbf{S}[k] \mathbf{S}[k]^{\top}
$$

It is possible to demonstrate that [22], [21]:

$$
\widehat{R}_{S S}=\sum_{k=i}^{K} \sigma_{S}^{2} \mathbf{G}\left(\theta_{T}, \phi_{T}\right) \mathbf{G}\left(\theta_{T}, \phi_{T}\right)^{\top}+\mathbf{I}
$$

where $\sigma_{S}^{2}=E\left|S[k]^{2}\right|$ is the SNR of the signal. Thus, applying the single value decomposition, the following matrices are obtained:

$$
\widehat{R}_{S S}=U S U^{*}
$$

The space spanned by the signal can be partitioned as $U=\left[U_{S}, U_{n}\right]$. The $N \times 1$ matrix $U_{S}$ is the signal subspace and contain the singular vector corresponding to the largest singular value, while the matrix $U_{n}$ is the signal null space and containing the $N-1$ singular vector corresponding to the noise singular values. Since $U$ is a unitary matrix, the signal and noise subspace are orthogonal, satisfying the equality $U_{S} \times U_{N}=0$. Accordingly, we can define a pseudo-spectrum

$$
\operatorname{PS}(\theta, \phi)=\frac{1}{\mathbf{G}(\theta, \phi) U_{n}}
$$

that exhibits a abrupt peak for the angles $(\theta, \phi)$ close to the true DoA, therefore the estimated DoA is formally identified with:

$$
\left(\widehat{\theta}_{T}, \widehat{\phi}_{T}\right)=\underset{\theta, \phi}{\operatorname{argmax}} \operatorname{PS}(\theta, \phi)
$$

The main drawback of this approach is the need to perform a search over the entire domain of the pseudo-spectrum, which correspond to the SBA gain space. The expected precision of the localization engine is related the angular resolution of the gain maps, therefore a trade-off between speed and precision is necessary.

As highlighted in Section III-C, the spatial multiplexing ability demonstrated by the node can be considered a rough localization algorithm. This fact can be conveniently employed to relax the search complexity, limiting the domain of argmax operator in eq. 8 to the pre-selected area individuated by the correspondent strongest signal of the vector $\mathbf{S}$, eventually after a mean operation. Referring to eq. 3 , the strongest beam can be identified even in the first phase of the data collection.

As a final note, classical MUSIC approaches are capable to deal only with a maximum number of node equal to the number of beams. The proposed approach, based on a time division scheme for the communication, can track a number of nodes which is limited only by the network capability and congestion, and it is fastest then the classical approach thanks to its preprocessor stage.

\section{EXPERIMENTAL VALIDATION}

In this section, the experimental validation of the system is presented. An intensive measurement campaign is conducted to validate the localization performance of the node. The accuracy of the DoA estimations have deep consequences on the performance of the positioning service performed by an eventual network of nodes. As previously stated, the network of anchors is thought to operate with a target node built with similar hardware but equipped only one antenna.

Three principal cuts for the link between the anchor and the target are evaluated. They are adequate to globally describe the behavior of the entire domain of the system, since the regular nature of the antenna arrangement. All the estimations are expressed in degree, for an immediate readability. 


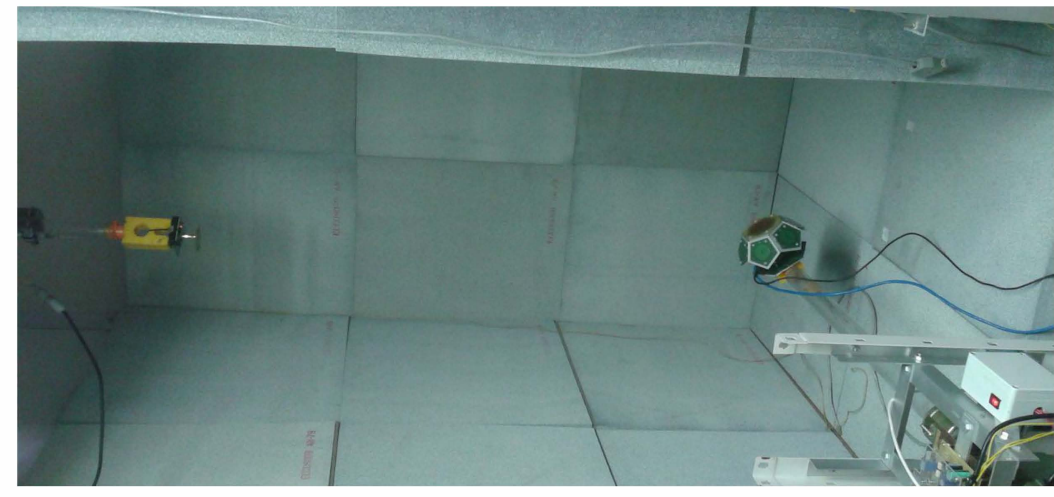

(a) Measurement setup

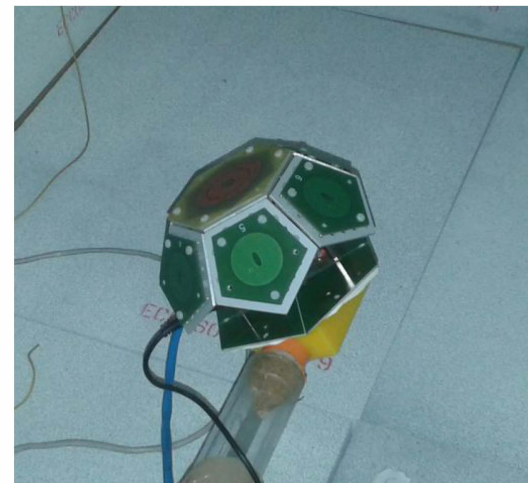

(b) Detail of the anchor

Fig. 11. Experimental semi-anechoic setup for the device characterization. On the left side of the figure 11(a), the target node is positioned on a fixed reference. On the right side, the anchor node is applied to the rotor used to scan the angular range of interest. In the particular of figure 11(b), the anchor arranged for measurement of the cut $\phi=120^{\circ}$ is shown.

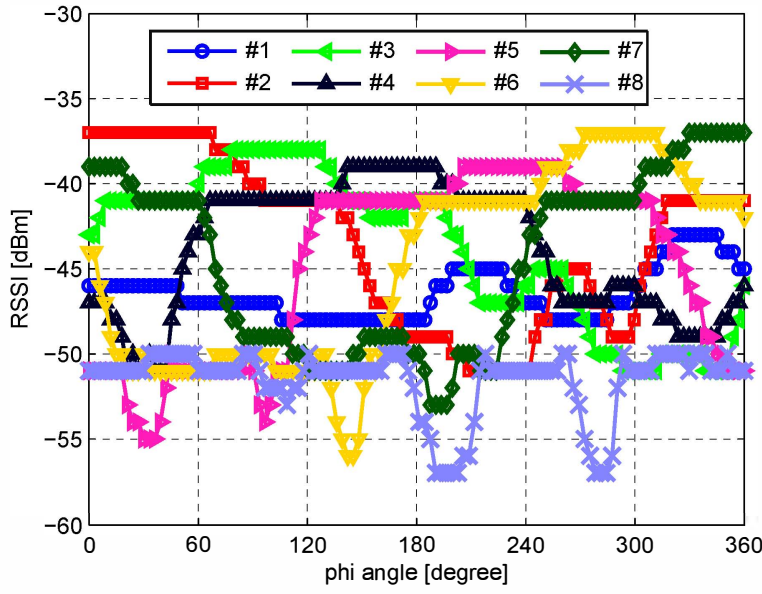

Fig. 12. Collected RSSI data for the cut $\theta=90$

\section{A. Localization for the cut $\theta=90^{\circ}$}

Fig. 12 shows the collected RSSI data in the first measurement cycle. 100 repetitions of 8-dimensional RSSI vectors are collected for each angle, and each 8-dim vector is the result of the mean of 5 samples at firmware level. The distance between the target node and the anchor is 1.25 meters and the target is equipped with an antenna similar to the to type I. It is believed that this antenna, whose pattern can cover half of an hemisphere without nulls, is adequate for the coverage of the target node. The chosen distance is adequate for far-field measurement, and it is of the same order of magnitude of the distances expected in the use of the proposed device. This measurement setup is similar to the one of the measurement of Fig. 9, with the target node orbiting around the anchor reference.

The RSSI data almost follows the same shape of the measured patterns, while affected by some non-idealities. Apart form the almost quantized nature, due to RSSI dB resolution, which emerges even after a 100 samples mean, the uniformity is lost due to the non-ideality of the SP8T switch, and due to the different loss of the micro cables connecting the switch to the antenna elements. Another source of error is the actual assembling of the node. The gain measurement were taken with the SBA shell removed from the mainboard.

Fig. 13 shows the results of the tuned-music algorithm to this first set of data. In particular, Fig. 13(b) shows the estimated $\phi$ angle. In an ideal condition, the estimated trace would describe the dashed straight line depicted in Fig. 13(b) along to the estimations.

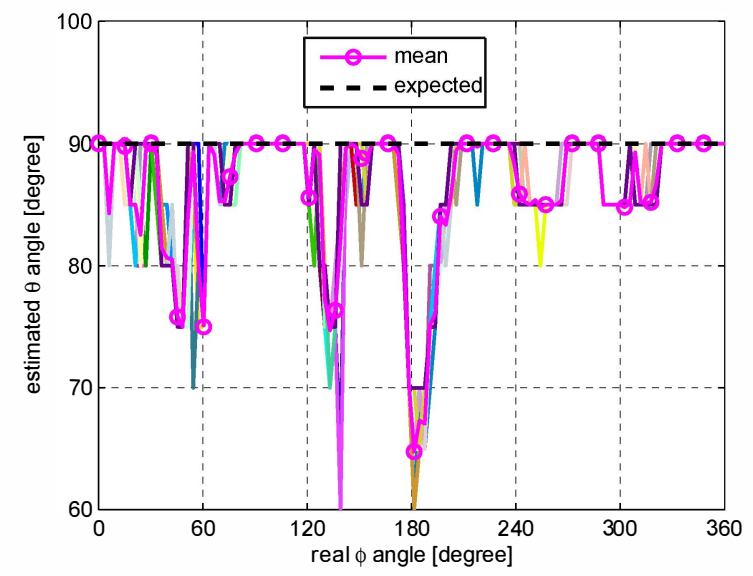

(a) Estimated $\theta$

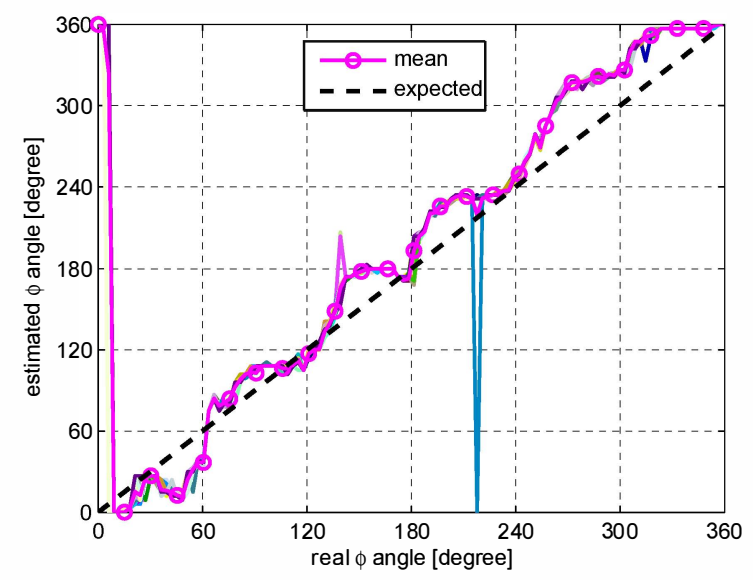

(b) Estimated $\phi$

Fig. 13. Estimated DoA from the data of Fig.12 
The agreement of the expected and obtained traces is very good, with a periodic behavior of the error, which seems to follow the periodic shape of the SBA seen from the equatorial cut. From the numerical data, is possible to calculate the mean $\phi$ error, $\epsilon_{\phi}=18.5^{\circ}$.

Fig. 13(a) depicts the estimated $\theta$ angle. Ideally, this angular value should be equal to $90^{\circ}$ for the entire $\phi$ turn. In this estimation, the maximum $90^{\circ}$ condition is forced by the definition of the gain map, since the $\theta$ variable extends from 0 to $90^{\circ}$, corresponding to pointing parallel to the ceiling of Fig. 2. While this is one of the worst condition for the $\theta$ estimation, since only one of the 8 beams is in an effective reaction condition ensuring trustful RSSI reading, the estimated mean $\epsilon_{\theta}$ is below $5^{\circ}$.

\section{B. Localization for the cut $\phi=0^{\circ} / 180^{\circ}$}

Another set of data is collected in the same operative condition. In this case, the measurement cut lies parallel to the $\theta$ variable, starting at $\phi=0^{\circ}$ and ending at $\phi=180^{\circ}$ in the reference of Fig. 8(a). In other words the target orbits from the south direction to the north passing through the polar direction $\theta=0^{\circ}$.

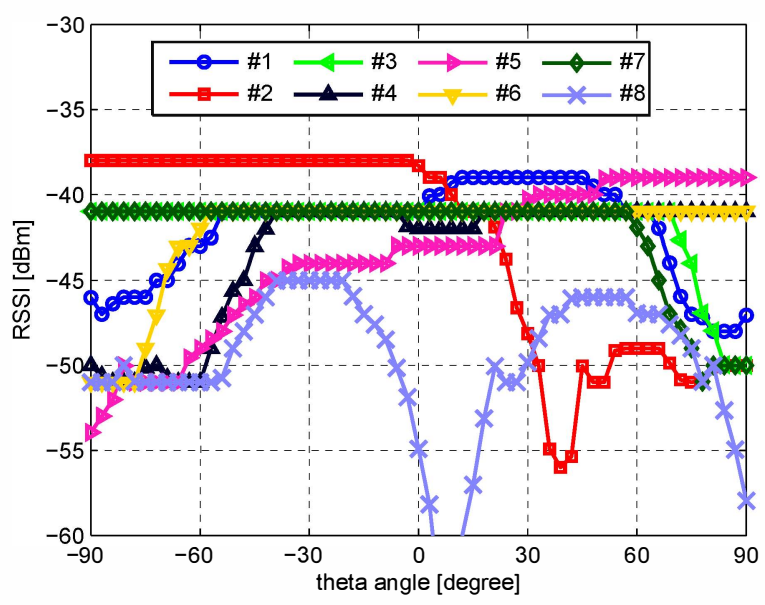

Fig. 14. Collected RSSI data for the cut $\phi=0^{\circ}$

Fig. 14 shows the mean collected data for this set of measurements, while Fig. 15 depicts the localization results. This time the $\theta$ coordinate of the target is supposed to describe a $V$ curve, starting at $\theta=90^{\circ}$ when $\phi=0^{\circ}$ - in front of element \#2 - passing the polar condition $\theta=0^{\circ}, \phi=0^{\circ}$ - in front of face $\# 1$ - to finish again at $\theta=90^{\circ}$ in the opposite direction - where face \#5 lies.

In Fig. 15(a) the V shape is clearly visible, even if a little distorted, with the cusp shape beyond the reference of the orbital abscissa. Looking at the corresponding collected data in Fig. 14, a similar biased behavior is present even in the RSSI traces, in particular considering the $\mathbf{S}_{1}$ and $\mathbf{S}_{8}$ in comparison with the corresponding traces in Fig. 10, therefore chances are that a misalignment in the measurement setup caused a shift in the angular reference.

In Fig. 15(b), the estimated $\phi$ is expected to describe a step shape, i.e. a constant value of $0^{\circ}$ along the negative $\theta$ semi-axis and a constant value of $180^{\circ}$ for the positive $\theta$ semi-axis. It is worthwhile that, for $\theta \approx 0^{\circ}$, the $\phi$ error is almost meaningless, since for $\theta=0^{\circ}$ the angle $\phi$ is undefined, and in a small range around it, even a $\phi$ phase shift of $180^{\circ}$ translates in a small absolute error when projecting the line of Bearing.

The calculated mean errors are $\epsilon_{\phi}=48^{\circ}$, and $\epsilon_{\theta}=13.5^{\circ}$, but with a manual re-alignment of the step-shaped $\phi$ curve, the estimated $\epsilon_{\phi}$ improves to $15^{\circ}$.

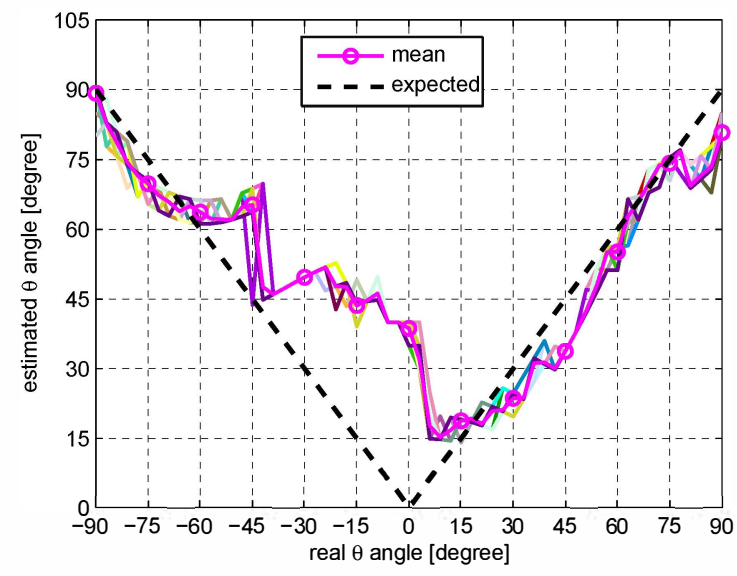

(a) Estimated $\theta$

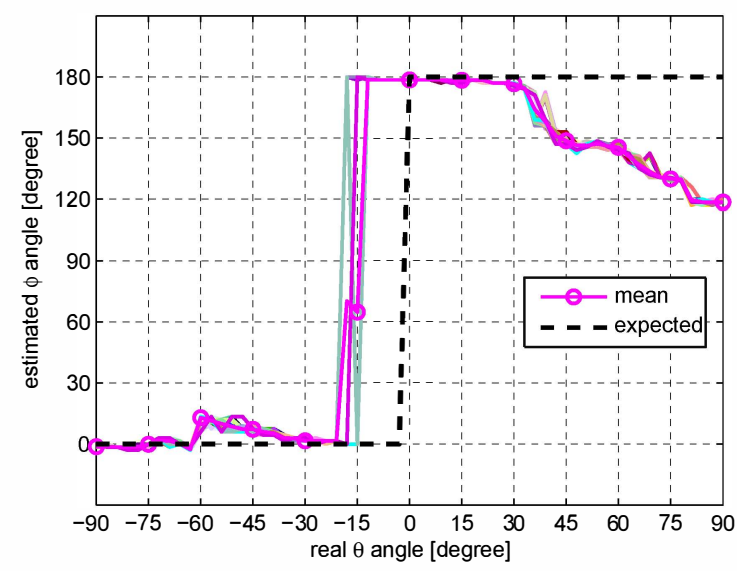

(b) Estimated $\phi$

Fig. 15. Estimated DoA from the data for the cut $\phi=0^{\circ}$, data of Fig. 14

\section{Localization for the cut $\phi=90^{\circ} / 270^{\circ}$}

The final set of data is collected with another the cut parallel to the $\theta$ variable, but starting at $\phi=90^{\circ}$ and ending at $\phi=$ $270^{\circ}$ (Fig. 16). This cut is orthogonal to the previous one, with the $\theta$ coordinate expected to describe again a $V$ curve, but starting from the edge between elements \#3 and \#4, and and ending in edge of elements \#6 and \#7. The mean collected data are shown in Fig. 16.

The results of the estimation algorithm are shown in Fig. 17, with Fig. 17(a) showing the $V$-cut of $\theta$. The cut shows a less defined cusp, but the monotone behavior is preserved.

Fig. 17(b) depicting with the step-shaped $\phi$ coordinate ranging from $90^{\circ}$ to $270^{\circ}$. The different performance respect to the previous case can be explained considering the different nature of this cut. In this case, a higher discrimination is 
provided by the edge nature of the cut itself. at $\phi=90^{\circ}$ two elements are expected to receive almost with the same RSSI intensity. This is a strong help to determines the exact $\phi$ variable, which is locally strongly influenced by the unbalance of $\mathbf{S}_{2}$ and $\mathbf{S}_{3}$. The same goes for the $\mathbf{S}_{5}$ and $\mathbf{S}_{6}$ on the other side. For a similar reason, the $\theta$ cut of Fig. 17(b) shows a better behavior of the same cut of Fig. 15(b).

The mean errors are $\epsilon_{\theta}=15^{\circ}, \epsilon_{\phi}=20^{\circ}$, but the latter drops to $14^{\circ}$ after a re-alignment such as in the previous section.

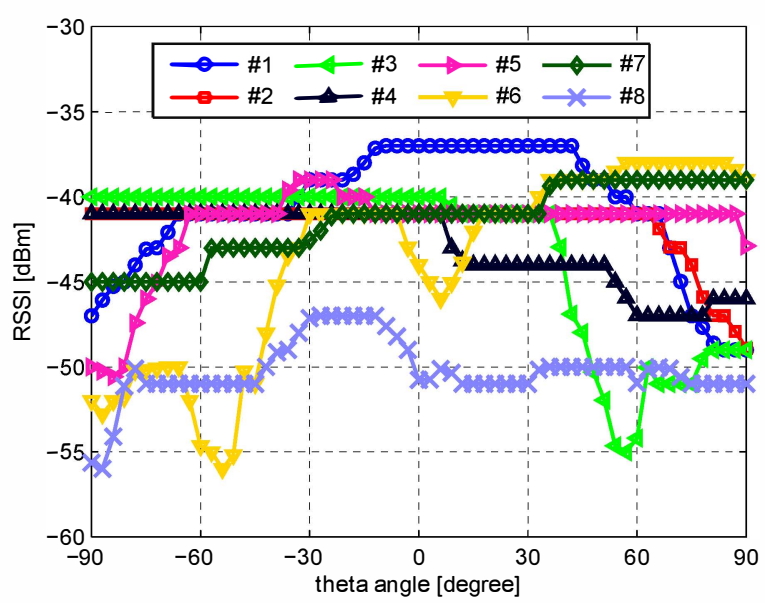

Fig. 16. Collected RSSI data for the cut $\phi=90^{\circ}$

Two important conclusions can be drown from the experimental measurements. The first one is the almost monotonic behavior of the estimated results. Each estimated DoA $\left(\widehat{\theta_{T}}, \widehat{\phi}_{T}\right)$ corresponds only to a single true DoA $\left(\theta_{T} \phi_{T}\right)$, i.e. the function mapping the estimated DoA to the true DoA is bijective. Therefore it is possible to elaborate the raw estimations in a post-processing stage. This possibility descends from the fact that the system shows almost systematic errors with very few random errors. A better accuracy can achieved inverting the unideal behavior of the response in order to linearize and regularize the estimation. This operation can be difficult if the gradient of the mapping function tends to zero, such for the cases in the border of Fig. 17(a).

A second observation derives from the fact that the gain map serving as input of the algorithm are synthesized by the measurements of the SBA in in a controlled setup. Apart from the importance of accurate interpolations of the missing data to avoid distortions in the rebuilt gain map, the real problem is that the maps taken in a controlled setup are different from the maps of the actual experimental environment. In fact, when placed in proximity of complex metallic objects, the gain pattern of the SBA is expected to change and deforms. Fortunately, if the distortion is below a critic amount, a possible improvement can obtained with a calibration procedure, as the one described in [23]. With this approach it is possible to tune some SBA gain parameters, eventually in the form of a matrix multiplier for the gain map, with a reduced set of RSSI measurements directly taken on the experimental site.

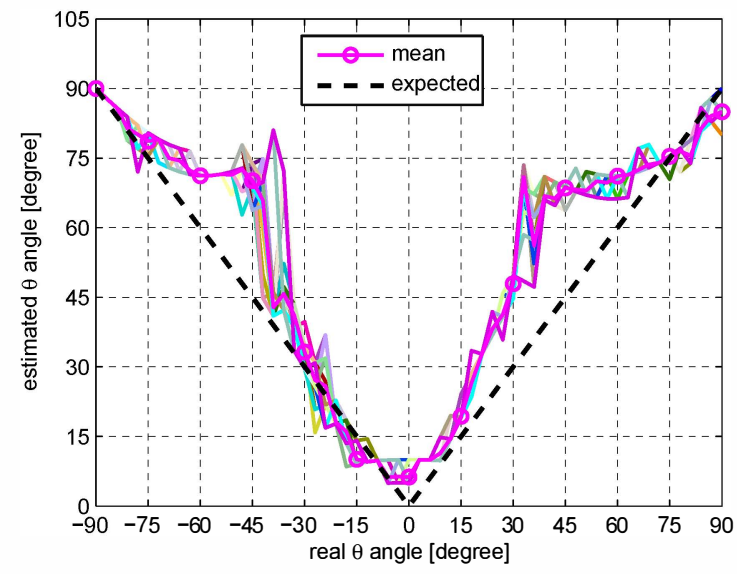

(a) Estimated $\theta$

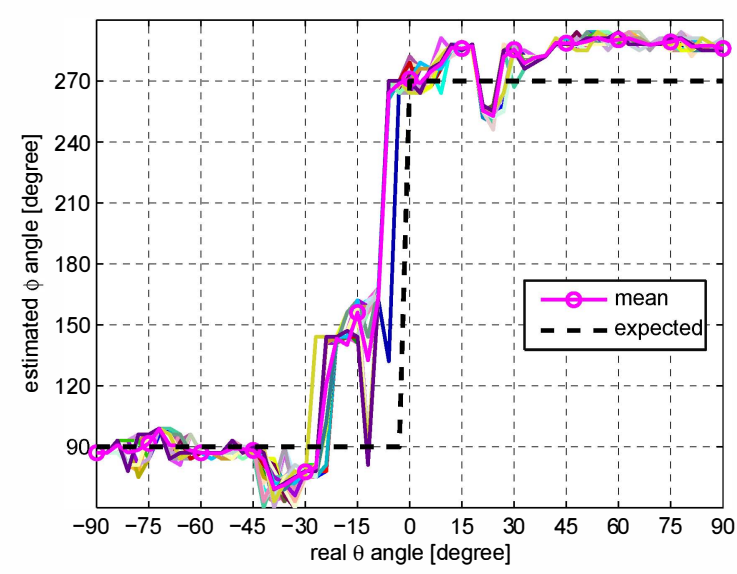

(b) Estimated $\phi$

Fig. 17. Estimated DoA from the data for the cut $\phi=90^{\circ}$, data of Fig. 16

\section{CONCLUSIONS}

A compact distributed system for localization in indoor environments was proposed. The system is based on smart anchor nodes, capable of WiFi connection, which operate as evolved and specialized access points.

Thanks to its smart Switched Beam Antenna system, each node is capable of selective spatial communications, and this is the enabling technology for the estimation of the Direction of Arrival of the radio messages sent by mobile nodes. This ability comes at very reduced cost and complexity, since the same architecture necessary for the WiFi communication is also used for the localization procedure. Conscious of this distributed ability, the nodes of a network are expected to collaborate, combining the collected DoA informations. From the set of DoA's referred to each anchor node reference, a system of lines of bearing is obtained, which finally resolves in the absolute position of the independent targets by the means of a triangulation strategy.

In this paper in particular, the single anchor node, core element of the network, was presented, designed, fabricated and validated. The node is built with standard COTS technology, and it is capable to perform DoA estimations operating on RSSI data which are automatically available during $\mathrm{WiFi}$ packet exchanges. The DoA estimation performance of the 
basic node shows mean errors around $15^{\circ}-20^{\circ}$ for both the $\theta$ and $\phi$ coordinates over the principal cuts describing the entire domain of interest. The reported performances are expected to improve with on-site calibration procedure, as suggested by the experimental tests.

When placed on the ceiling, the expected radio signal distribution of the node is also capable of cumulative space-filling coverage. In addition to the exhibited DoA performance, this communication feature candidates the node as the perfect unit of a scalable distributed low-cost localization system thought to operate in harmony with common indoor WiFi. The actual performances in terms of metric error of a network of nodes are obviously functions of the node DoA estimation error, but they are also strongly dependent on the actual number and arrangement of nodes. The network density and geometry can have deep consequences on the dilution of precision of the system.

A detailed description and characterization of the localization performance of the network of the proposed nodes is the object of the next investigation of the authors.

\section{REFERENCES}

[1] S. Maddio, A. Cidronali, and G. Manes, "Smart antennas for directionof-arrival indoor positioning applications," in Handbook of Position Location: Theory, Practice, and Advances. Wiley Online Library, 2011, pp. 319-355.

[2] L. Bencini, S. Maddio, G. Collodi, D. Di Palma, G. Manes, and A. Manes, "Development of wireless sensor networks for agricultural monitoring," in Smart Sensing Technology for Agriculture and Environmental Monitoring. Springer, 2012, pp. 157-186.

[3] S. Maddio, A. Cidronali, and G. Manes, "Rssi/doa based positioning systems for wireless sensor network," in New Approach of Indoor and Outdoor Localization Systems, 2012.

[4] L. Bencini, G. Collodi, D. Di Palma, G. Manes, and A. Manes, "An energy efficient cross layer solution based on smart antennas for wireless sensor network applications," in Sensor Technologies and Applications (SENSORCOMM), 2010 Fourth International Conference on. IEEE, 2010, pp. 232-237.

[5] R. Ramanathan, J. Redi, C. Santivanez, D. Wiggins, and S. Polit, "Ad hoc networking with directional antennas: a complete system solution," Selected Areas in Communications, IEEE Journal on, vol. 23, no. 3, pp. 496-506, 2005.

[6] C.-C. Lin, S.-Y. Wang, and T.-W. Hsu, "On the performances of ieee 802.16 (d) mesh cds-mode networks using single-switched-beam antennas," Computer Networks, vol. 56, no. 4, pp. 1402-1423, 2012.

[7] A. Cidronali, S. Maddio, G. Giorgetti, and G. Manes, "Analysis and performance of a smart antenna for 2.45-ghz single-anchor indoor positioning," Microwave Theory and Techniques, IEEE Transactions on, vol. 58, no. 1, pp. 21-31, 2010.

[8] T. Rappaport and D. Hawbaker, "Wide-band microwave propagation parameters using circular and linear polarized antennas for indoor wireless channels," Communications, IEEE Transactions on, vol. 40, no. 2, pp. 240-245, 1992.

[9] R. Szumny, K. Kurek, and J. Modelski, "Attenuation of multipath components using directional antennas and circular polarization for indoor wireless positioning systems," in Radar Conference, 2007. EuRAD 2007. European. IEEE, 2007, pp. 401-404.

[10] G. Giorgetti, S. Maddio, A. Cidronali, S. Gupta, and G. Manes, "Switched beam antenna design principles for angle of arrival estimation," in Wireless Technology Conference, 2009. EuWIT 2009. European. IEEE, 2009, pp. 5-8.

[11] K. Carver and J. Mink, "Microstrip antenna technology," Antennas and Propagation, IEEE Transactions on [legacy, pre-1988], vol. 29, no. 1, pp. 2-24, 1981.

[12] R. Garg, Microstrip Antenna Design Handbook. Artech House, 2001

[13] J. James, C. Wood, and P. Hall, Microstrip Antenna Theory and Design. Iet, 1986.
[14] S. Maddio, A. Cidronali, and G. Manes, "A new design method for single-feed circular polarization microstrip antenna with an arbitrary impedance matching condition," IEEE Transactions on Antennas and Propagation, vol. 59, no. 2, pp. 379-389, 2011.

[15] S. Maddio, A. Cidronali, I. Magrini, and G. Manes, "A design method for single-feed wideband microstrip patch antenna for switchable circular polarization," Microwave Conference, 2007. European, pp. 262-265, 2007.

[16] S. Maddio, A. Cidronali, and G. Manes, "An azimuth of arrival detector based on a compact complementary antenna system," in Wireless Technology Conference (EuWIT), 2010 European. IEEE, 2010, pp. 249-252.

[17] Y. Chen, J. Yang, W. Trappe, and R. P. Martin, "Impact of anchor placement and anchor selection on localization accuracy," in Handbook of Position Location: Theory, Practice, and Advances. Wiley Online Library, 2011, pp. 425-455.

[18] G. Giorgetti, S. Maddio, A. Cidronali, S. Gupta, and G. Manes, "Switched beam antenna design principles for angle of arrival estimation."

[19] T. G. Vasiliadis, A. G. Dimitriou, and G. D. Sergiadis, "A novel technique for the approximation of 3-d antenna radiation patterns," Antennas and Propagation, IEEE Transactions on, vol. 53, no. 7, pp. 2212-2219, 2005.

[20] W. Araujo Lopes, G. Glionna, and M. S. De Alencar, "Generation of 3d radiation patterns: a geometrical approach," in Vehicular Technology Conference, 2002. VTC Spring 2002. IEEE 55th, vol. 2. IEEE, 2002, pp. 741-744.

[21] H. Krim and M. Viberg, "Two decades of array signal processing research: the parametric approach," Signal Processing Magazine, IEEE, vol. 13 , no. 4, pp. 67-94, 1996.

[22] R. Schmidt, "Multiple emitter location and signal parameter estimation,' Antennas and Propagation, IEEE Transactions on, vol. 34, no. 3, pp. 276-280, 1986.

[23] S. Maddio, A. Cidronali, G. Giorgetti, and G. Manes, "Calibration of a $2.45 \mathrm{ghz}$ indoor direction of arrival system based on unknown antenna gain," in Radar Conference (EuRAD), 2010 European. IEEE, 2010, pp. $77-80$. 